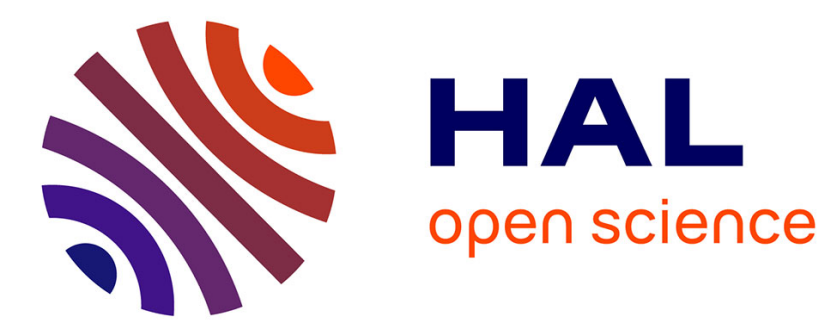

\title{
Post and pre-initialized stopwatch Petri Nets
}

\author{
Adib Allahham, Hassane Alla
}

\section{To cite this version:}

Adib Allahham, Hassane Alla. Post and pre-initialized stopwatch Petri Nets. DCDS 2007 - 1st IFAC Workshop on Dependable Control of Discrete-event Systems, Jun 2007, Cachan, France. pp.69-74. hal-00157454

\section{HAL Id: hal-00157454 https://hal.science/hal-00157454}

Submitted on 26 Jun 2007

HAL is a multi-disciplinary open access archive for the deposit and dissemination of scientific research documents, whether they are published or not. The documents may come from teaching and research institutions in France or abroad, or from public or private research centers.
L'archive ouverte pluridisciplinaire HAL, est destinée au dépôt et à la diffusion de documents scientifiques de niveau recherche, publiés ou non, émanant des établissements d'enseignement et de recherche français ou étrangers, des laboratoires publics ou privés. 


\title{
POST AND PRE-INITIALIZED STOPWATCH PETRI NETS
}

\author{
Adib Allahham* Hassane Alla* \\ * GIPSA-Lab, Dpartement d'Automatique - BP 46 \\ 38402 Saint Martin d'Hères, France \\ Email: \{Adib.al-lahham,hassane.alla\}@inpg.fr
}

\begin{abstract}
A modeling paradigm called Post and Pre-initialized Stopwatch Petri Nets $(S W P N)$ is introduced. It extends Time Petri Nets $T P N$ to the concept of stopwatch with an original mechanism of stopwatches reset. SWPN makes this reset dependent on the firing of the corresponding transitions. The resulting model permits natural description of so-called preemption-resume behavior. We give the formal semantics of $S W P N$ as a timed transition system. We propose also a method for its analysis consisting in the computation of its equivalent stopwatch automaton $S W A$. The advantage of Petri Nets for modeling the complex system in concise way is combined with the power analysis of SWA.Copyright (c) 2007 IFAC
\end{abstract}

Keywords: T-time Petri Net, Stopwatch automata, timing analysis.

\section{INTRODUCTION}

Interruptible systems are often found in real-time systems that are typically composed of several tasks that interact. The behavior of these systems is so-called preemption-resume behavior where the some of its tasks are suspended and resumed latter. An important problem consists in ensuring that the tasks can be executed in such a way that they respect the constraints they are subjected to, as deadlines for example. So the influence of interruptions must be considered in modeling and verifying these systems. This requires to describe the suspension and resuming of tasks. To fulfill these needs, several models based on the notion of Stopwatch (a clock that can be stopped and resumed) have been proposed in the literature. One can find stopwatch extensions of classical dense time model: Timed Automata $T A$ and time Petri Nets TPNs. Among the extensions of the timed automata, the Stopwatch Automata $S W A$ (Cassez and Larsen (2000)) are defined as a subclass of the linear hybrid automata. In this model the increasing clock rate can be switched between (1) and (0) in order to express the progression or the suspension of a task across different logical locations. Petri Nets are another widely used mode for real-time system. They permit generally both, to facilitate the description of conditions, and to enhance the readability of the specifications which is not the case for direct modeling in $S W A$. Therefore, several efforts have been done to model the preemptive behavior for scheduling purposes by using some extensions of Time Petri Nets TPN. Lime and Roux (2004) proposed an extension called Scheduling Extended Time Petri Nets that consists in mapping into a PN model the way the different schedulers of a system activate or suspend tasks. For a fixed priority scheduling policy, Scheduling-TPNs introduce two new attributes associated with each place that respectively represent allocation and priority. Bucci et al. (2004) proposed a similar model: Preemptive Time Petri Nets. The two attributes are associated with transitions instead of places. Roux and Lime (2004) defined Time Petri Nets with Inhibitor Hyperarcs as an extension of $T-T P N$. The stopwatch 
associated with a transition can be reset, stopped and started by using classical arcs and branch inhibitor hyperarcs. Firing of a transition may be interrupted if there is a nonempty place connected to this transition by an inhibitor arc.

The Petri net extensions mentioned above introduce in the model either the priority or the inhibitor arcs for modeling the suspension and resuming. These attributes increase the difficulties of modeling complex systems as manufacturing systems. The need of a simple modeling tool to model preemption-resume behavior motivates us to propose the Post and Pre-initialized Stopwatch Petri nets. This model is referred in the sequel as Stopwatch Petri Nets(SWPN,s for short). An important contribution in our proposed model $S W P N$ is the notion of pre-initialization and postinitialization of the clocks. In a $T P N$, only the pre-initialization is used. The clocks are initialized when its transitions are newly enabled. In $S W P N$, we introduce the post-initialization where the clocks are initialized when their associated transitions are fired. This mechanism of initialization of the variables associated with transitions is used in (Bobbio et al. (2000)) to widen the field of applicability of the stochastic petri nets, and thus this allows to model different preemption policies. The key difference with respect to our formulation is that between TPN and stochastic PN (there is no actual conflict in a stochastic Petri nets. For a detailed presentation of timed conflicts see (David and Alla (2005))). In turn, this difference reflects to the analysis method.

We tackle the reachability problem for the $S W P N$ which it is, as all the extension of TPN mentioned above, undecidable. Our method for timing analysis is based on translation the $S W P N$ into an equivalent $S W A$. Then, a forward analysis will be applied on the resulting $S W A$ using PHAVer model-checker (Frehse (2005)). Thus, we bring the techniques used in PHAVer to force the termination, when the state space is not computable.

In Section 2 the $S W P N$ model, its syntax and semantics are presented. The translation algorithm into a $S W A$ is described in Section 3. Section 4 illustrates the proposed model and its timing analysis method by an example.

\section{STOPWATCH PETRI NETS}

We define the Stopwatch Petri Nets SWPNs which extends the basic model of T-time Petri Nets (Berthomieu and Diaz (1991)), to the concept of stopwatch with an original mechanism of stopwatches reset. It makes the reset of some timed transition dependent on the firing of the transitions (notion of post-initialization). In $S W P N$, there are two types of transitions: interruptible and non-interruptible transitions. The

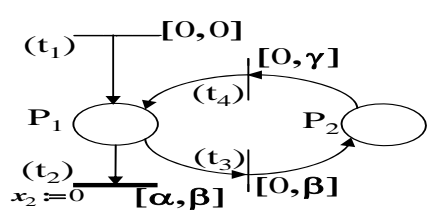

Fig. 1. $S W P N$ of an interruptible task

firing of an interruptible transition resets the associated stopwatch, while the firing of another noninterruptible transition making the interruptible transition not enabled, suspends the progress of the time to fire which is then resumed when the transition is enabled again.

Example: Suppose there is an interruptible task having duration of execution $[\alpha, \beta]$ (without the durations of the interruption). After each interruption, the task resumes in the same place when it was interrupted. The interruption can occur at any instant during the task's execution. The maximal duration of each interruption is $\gamma \in \mathbb{R}^{+}$. The proposed $S W P N$ models this behavior as shown in Figure 1. Place $P_{1}$ represents the execution of the task while place $P_{2}$ represents the interrupted state of task. Transitions $t_{3}$ and $t_{4}$ represent respectively the occurrence of interruption and resuming of the task. Transition $t_{2}$ which is an interruptible transition represents the task's execution. It is associated with stopwatch $x_{2}$. A graphical representation to distinguish between interruptible and non-interruptible transitions is to draw the former one in bold and to associate it by the assignment $x_{2}:=0$. When an interrupt occurs, the token emerges from $P_{1}$ into $P_{2}$ and $x_{2}$ becomes inactive. When the interruption ends, transition $t_{4}$ fires, the task resumes in the same place where it was interrupted and $x_{2}$ comes back active and restores its saved value when the interruption happened. Firing $t_{2}$ resets the stopwatch $x_{2}$. At the initial marking, all the stopwatches of $S W P N$ model reset to 0 .

Definition 1. A Stopwatch Petri Nets is a tuple $\left\langle P, T, \bullet(),.(.)^{\bullet}, M_{0}, I_{s}\right\rangle$, where:

- $\mathrm{P}$ is a non-empty finite set of places;

- $\mathrm{T}$ is a non-empty finite set of transitions. The set $T=T_{i n t} \cup T_{n o-i n t}$ is composed of two disjoints sets: the interruptible and noninterruptible transitions;

- $($.$) and (.) \bullet$ are respectively the backward and forward incidence function;

- $M_{0} \in \mathbb{N}^{c a r d|P|}$ is the initial marking of the net, associating with each place $p$ a natural number of tokens;

- $I_{s}$ associates with each transition $t_{i}$ a firing interval delimited by an earliest firing time $\operatorname{EFT}\left(t_{i}\right) \in \mathbb{R}^{+}$and a latest firing time $\operatorname{LFT}\left(t_{i}\right) \in \mathbb{R}^{+} \cup\{\infty\}$. 


\subsection{Semantics:}

A marking $M$ of the net is an element of $\mathbb{N}^{\operatorname{card}|P|}$ such that $\forall p \in P, M(p)$ is the number of token in the place $p$. A transition $t_{i}$ is to said to enabled by marking $M$ if $M \geq{ }^{\bullet} t$ and denoted by $t_{i} \in \operatorname{enabled}(M)$.

Let be $v\left(t_{i}\right)$ represents the values of the stopwatch associated to $t_{i} \cdot v\left(t_{i}\right)$ is a mapping $v \in$ $\left(R^{+}\right)^{\operatorname{card}|T|}$ such that:

- $\forall t_{i} \in T_{i n t}, v\left(t_{i}\right)$ is the time elapsed since $t_{i}$ was first enabled and during which $t_{i}$ remained enabled. $t_{i}$ is first enabled when it is enabled and its value $v\left(t_{i}\right)=0$. Remind that $v\left(t_{i}\right) \leftarrow 0$ at each time $t_{i}$ fires;

- $\forall t_{i} \in T_{n o-i n t}, v\left(t_{i}\right)$ is the time elapsed since $t_{i}$ was last enabled.

A transition $t_{i} \in T_{\text {int }}$ is said to be suspended by the marking $M$ and denoted by $\operatorname{susp}(M)$ if :

$t_{i} \in T_{\text {int }}: t_{i} \in \operatorname{susp}(M)$ if $\bullet\left(t_{i}\right)>M \wedge v\left(t_{i}\right)>0 . \square$

A transition $t_{i}$ is said to be firable by marking $M$ and denoted by $t_{i} \in$ firable $(M)$ if:

$t_{i} \in \operatorname{enabled}(M) \wedge \operatorname{EFT}\left(t_{i}\right) \leq v\left(t_{i}\right) \leq L F T\left(t_{i}\right) . \square$ A transition $t_{i} \in T_{n o-i n t}$ is said to be newly enabled by firing the transition $t_{k}$ from the marking $M$ and denoted by $\uparrow$ enabled $\left(t_{i}, M, t_{k}\right)$ if: $\left(t_{i} \notin \operatorname{enabled}(M) \vee\left(t_{i}=t_{k}\right)\right) \wedge\left(\bullet\left(t_{i}\right) \leq M-\right.$ $\left.\bullet\left(t_{k}\right)+\left(t_{k}\right)^{\bullet}\right)$.

Notion of pre-initialization: The transition $t_{i}$ is called Pre-initialized, if we initialize it before its using. The concept of Pre-initialization is possible when $t_{i}$ is newly enabled, i.e, $t_{i} \in$ $\uparrow \operatorname{enabled}\left(t_{i}, M, t_{k}\right)$.

Notion of Post-initialization: The transition $t_{i}$ is called Post-initialized, if we initialize it after its using. The concept of Post-initialisation is possible only if $t_{i} \in T_{\text {int }}$.

The semantics of $S W P N$ as Timed Transition Systems (TTS) are defined as follows.

Definition 2. (Semantics of a $S W P N$ ) The semantics of a Stopwatch Petri Net $N$ is defined as a $T T S S_{N}=\left(Q, q_{0}, \rightarrow\right)$ such that:

- $Q=\mathbb{N}^{p} \times\left(\mathbb{R}^{+}\right)^{\operatorname{card}|T|}$.

- $q_{0}=\left(M_{0}, \overline{0}\right)$. In this state, the value of all the stopwatches is reset.

- $\rightarrow \in Q \times(T \cup \mathbb{R}) \times Q$ is the transition relation including a continuous transition relation and a discrete transition relation.

- the continuous transition relation is defined $\forall d \in \mathbb{R}^{+}$by:

$$
\begin{aligned}
& (M, v) \stackrel{d}{\longrightarrow}\left(M, v^{\prime}\right) \text { iff } \forall t_{i} \in T \\
& \left\{\begin{array}{l}
v^{\prime}\left(t_{i}\right)=\left\{\begin{array}{l}
v\left(t_{i}\right)+d \text { if } t_{i} \in \operatorname{enabled}(M) \\
v\left(t_{i}\right) \text { Otherwise }
\end{array}\right. \\
M \geq \bullet\left(t_{i}\right) \Rightarrow v^{\prime} \leq L F T\left(t_{i}\right)
\end{array}\right.
\end{aligned}
$$

- the discrete transition relation is defined $\forall t_{i} \in T$ by:

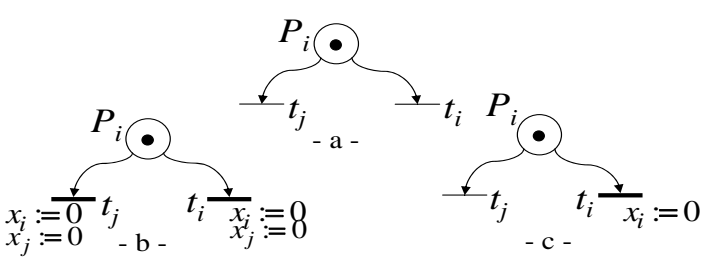

Fig. 2. Stopwatch reset in different conflict cases

$$
\begin{aligned}
& (M, v) \stackrel{t_{i}}{\longrightarrow}\left(M^{\prime}, v^{\prime}\right) \text { iff } \forall t_{i} \in T
\end{aligned}
$$

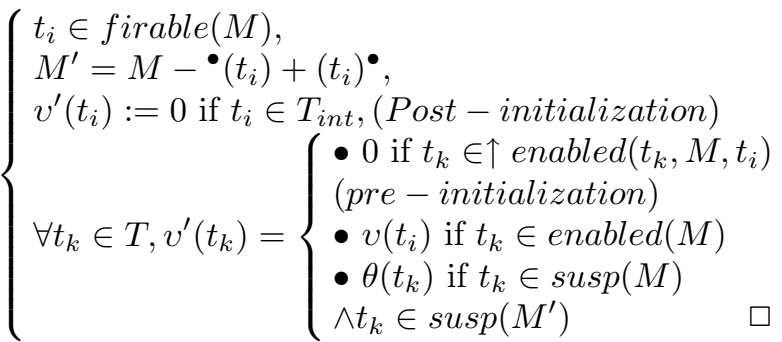

In discrete transition relation, the value $v^{\prime}$ of any transition in marking $M^{\prime}$ is different for persistent-progressing transitions and persistentsuspended transition. For the first one, it represents the time elapsed in the previous state while for the second one $v^{\prime}$ represents the value of the stopwatch when it was suspended in a previous state (at the value $\theta\left(t_{k}\right)$ ), remains frozen in the previous state characterized by the marking $M$ and stays frozen in the current state $M^{\prime}$.

\section{- Stopwatches reset in conflict case}

Figure 2 represents parts of $S W P N$. There is an effective conflict, namely $\left\langle P_{i},\left\{t_{i}, t_{j}\right\}\right\rangle$.

With regards to the reset of stopwatches associated with the transitions affected by a conflict, we distinguish the following cases according to the transitions types in conflict. The stopwatches which are initialized by a transition firing are represented by a set of assignments $x_{i}:=0$ shown close to this fired transition:

- $t_{i}, t_{j} \in T_{n o-i n t}$. This is the general behavior of T-Time Petri Nets (Fig.2.a).

- $t_{i}, t_{j} \in T_{\text {int }}$. The two stopwatches associated with $t_{i}$ and $t_{j}$ will be reset by firing one of the two transitions (Fig.2.b).

- $t_{i} \in T_{\text {int }}$ and $t_{j} \in T_{n o-i n t}$. Only the stopwatch associated with $t_{i}$ will be reset by its firing and the other will be reset when the corresponding transition is newly enabled (Fig. 2.c).

\subsection{Timing Analysis of $S W P N$}

In $T P N$, if the limits of the timed intervals associated to the transitions are rational numbers, and if the associated $P N$ is bounded then the $T P N$ is bounded. Outside of precedent sufficient boundedness conditions, only the computation of the state space allows to determine if a $S W P N$ is bounded or not. Techniques for reducing the infinite state space to a finite one are necessary. 
Thus, we are interested in bounded $S W P N$ for which we propose a technique for timing analysis. The values of stopwatches in a state of a $S W P N$ can be described by a complex non-convex polyhedron. So, computing its state space leads to polyhedra of increasing complexity and the number of polyhedra may grows without bounds and the reachability algorithm does not terminate. For that, we propose firstly to translate the $S W P N$ to an equivalent stopwatch automaton. Then, thanks to the hybrid systems analysis tools such as model-checker PHAVer (Frehse (2005)), the manipulation of these polyhedron is possible. In PHAVer, if the invariants of the automaton is bounded, then the analysis termination can be forced by using the simplification techniques of a complex polyhedra. The simplification is done in a strictly conservative approach of the reachable set of states.

\section{FROM STOPWATCH PETRI NET TO STOPWATCH AUTOMATA}

We aim to translate a $S W P N$ into a $S W A$ in order to perform a timing analysis of this $S W P N$, using efficient analysis $S W A$ techniques and tools.

\subsection{Stopwatch Automata}

The stopwatch automata is basically defined as a class of linear hybrid automaton where the time derivative of a variable in a location can be either 0 or 1 (Cassez and Larsen (2000)).

Definition 3. A Stopwatch automaton is a 7-tuple $\left(L, l_{0}, X, \Sigma, A, I, D i f\right)$ where

- $L$ is a finite set of locations,

- $l_{0}$ is the initial location,

- $X$ is a finite set of positive real-valued stopwatches,

- $\Sigma$ is a finite set of labels,

- $A \subset L \times C(X) \times \sigma \times 2^{X} \times L$ is a finite set of arcs. $a=\left(l, \delta, t, R, l^{\prime}\right) \in A$ is the arc between the locations $l$ and $l^{\prime}$, with the guard $\delta$, the label $t$ and the set of stopwatches to reset $R$. $C(X)$ is the set of constraints over $X$.

- $I \in C(X)^{L}$ maps an invariant to each location,

- Dif $\in\left(\{0,1\}^{X}\right)^{L}$ maps an activity to each location, $\dot{X}$ being the set of derivatives of the stopwatches w.r.t time. $\dot{X}=\operatorname{Dif}(l)(x)_{x \in X}$. Given a location $l$ and a clock $x$, we will denote $\operatorname{Dif}(l)(x)_{x \in X}=\{0,1\}$.

\subsection{Reachability techniques analysis for SWA}

A state of the $S W A$ is a pair $(L, E)$ where $L$ is a location of $S W A$ and $E$ is a polyhedron representing its timed state space. There are two types of evolutions from a state $(L, E)$, namely the continuous evolution by letting the time progress and the discrete evolution by firing an arc. Accordingly, there are two types of successors: continuous-successors denoted by $s u c c_{t}$ and discrete-successors denoted by succ $_{d}$. The interested readers can refer to (Alur et al. (1995)) for more details.

\subsection{A Forward Algorithm to Compute the State Space of a bounded SWPN}

The proposed method in this paper is an adaptation of the region based method for Timed $\mathrm{Au}-$ tomaton. The algorithm starts from the initial state and explores all possible evolutions of the $S W P N$ by firing transitions or by elapsing a certain amount of time.

3.3.1. Labeling of marking graph: Let $G$ be the marking graph of the $S W P N$. It can be easily be labeled to generate a Stopwatch automaton $S W A$ bisimilar to the $S W P N$.

The pair $G=(M, A)$ is composed of:

- $M$ is the set of possible markings of the $S W P N$ : $M_{0}, \ldots, M_{p}$,

- $A$ is the set of arcs between the nodes of the marking graph : $a_{0}, \ldots, a_{q}$.

The Stopwatch Automaton will be obtained by associating with each marking the differential equations that express the dynamics of stopwatches in this state (marking), and an invariant. Each arc between two reachable markings are associated with a guard and some clocks assignments. Each of these arcs corresponds to firing an enabled transition of the $S W P N$.

- Dynamic of stopwatches: A set of differential equations in the form $x=c$ where $c=\{0,1\}$ is associated with each marking $M_{k}$.

$\forall t_{i} \in \operatorname{enabled}\left(M_{k}\right): x_{i}=1$ and $\forall t_{j} \in$ suspended $\left(M_{k}\right): x_{j}=0$. The inactive transitions are not considered $\left(t_{m}\right.$ is inactive if if $\left(t_{m} \in T_{\text {int }} \wedge\right.$ $\left.t_{m} \notin \operatorname{enabled}\left(M_{k}\right) \wedge v\left(t_{m}\right)=x_{m}=0\right)$ or $\left(t_{m} \in\right.$ $T_{\text {no-int }} \wedge t_{m} \notin$ enabled $\left(M_{k}\right)$.

- Invariant: An invariant is associated with each marking $M_{k}$. By construction, in each marking, only the possible evolution of time is computed. In other words, only the active or suspended stopwatches will be represented in each location. Let $X_{k}$ be the set of stopwatches associated with the enabled and suspended transitions for the marking $M_{k}$ of the $S W P N$. Then, the invariant associated with $M_{k}$ is defined by:

$I\left(M_{k}\right)=\left\{x_{i} \leq \operatorname{LFT}\left(t_{i}\right) \mid t_{i} \in \operatorname{enabled}\left(M_{k}\right)\right.$ or $\left.\operatorname{susp}\left(M_{k}\right)\right\}$.

- Guard: Each arc $a_{k}$ of the graph $G$ corresponds to the firing of a transition $t_{i}$ in $S W P N$. Then, we label $a_{k}$ by: 
$\square$ the action name $t_{i}$

the guard: $\operatorname{EFT}\left(t_{i}\right) \leq x_{i} \leq \operatorname{LFT}\left(t_{i}\right)$,

the clocks assignments: $x_{k} \leftarrow 0$ for all clocks $x_{k}$ associated with a newly enabled transition $t_{k}$ where $t_{k} \in T_{\text {non-int }}$ (Pre-initialization). If the fired transition $t_{i} \in T_{i n t}$, we associated also the clock assignment $x_{i} \leftarrow 0$ (Post-initialization). If $t_{i} \in T_{\text {int }}$ is in an effective conflict with $t_{j} \in T_{\text {int }}$ then we add $x_{j} \leftarrow 0$.

$S W A_{G}$ denotes the stopwatch automaton obtained from labeling the marking graph. In this automaton, we denote each location according to its corresponding marking, i.e. $L_{0} \ldots . L_{k}$ correspond $M_{0}, \ldots . M_{k}$.

\section{- The Algorithm for one iteration:}

The algorithm proposed is based on the forward analysis to find the reachable location of $S W A_{G}$. These reachable locations are accumulated in the pile Reach. At the initial state, Reach $=\left\{L_{0}\right\}$.

The computation of the reachable states from $L_{0}$ and the polyhedral $E_{0}^{e}$ containing the values of stopwatches at the entry of $L_{0}$ is done as follows:

- Compute the possible evolution of time according to the active stopwatches in $L_{0}$ or the values of stopwatches for which $L_{0}$ could exist, i.e. values of stopwatches must not be greater than the latest firing time of enabled transitions: $E_{0}=\operatorname{Succ}_{t}\left(E_{0}^{e}\right)$.

- Determine the firable arcs which leaves $L_{0}$. $a_{0, k}$ is the arc which leaves $L_{0}$ to $L_{k} \cdot a_{0, k}$ which is associated with the label $t_{i}$ is firable if $E_{0} \cap\left\{E F T\left(t_{i}\right) \leq x_{i} \leq E F T\left(t_{i}\right)\right\}$ is a non empty-polyhedron. Then, Update the set Reach by adding $L_{k}$ : Reach $=\left\{L_{0}, L_{k}\right\}$.

- For each firable arc $a_{0, k}$ leading to a marking $L_{k}$, compute the values at the entering of $L_{k}$ :

$$
\begin{gathered}
S_{0, k}=\operatorname{Succ}_{d}\left(E_{0}\right) \\
E_{k}^{e}=S_{0, k} \wedge\left[X_{e}:=0\right]
\end{gathered}
$$

where $X_{e}$ is the set of stopwatches annotated with the affectation of $a_{0, k} . E_{k}^{e}$ is a polyhedron for which the new marking $L_{k}$ is reachable.

- Compute the possible evolution of time for which $L_{k}$ could exist: $E_{k}=\operatorname{Succ}_{t}\left(E_{k}^{e}\right)$.

\subsection{Reachable stopwatch automaton $S W A_{\text {Reach }}$}

The obtained stopwatch automaton by applying the forward analysis to $S W A_{G}$ is defined as follows:

Definition 4. $\bullet L=$ Reach $=\left\{L_{0}, \ldots \ldots \ldots, L_{k}\right\}$ is the set locations, i.e. the set of reachable marking of $S W P N . L_{0}=M_{0}$ is the initial marking,

- $X=\left\{x_{1}, x_{2}, \ldots \ldots, x_{q}\right\}$ is the set of stopwatches, i.e. the set of all stopwatches associated with the transitions,

- $\Sigma=\left\{t_{1}, \ldots . ., t_{q}\right\}$ is the set of labels, i.e. the transitions of $S W P N$,

- $A \subset L \times C(X) \times \Sigma \times 2^{C} \times L$ is the finite set of firable arcs between the reachable locations,

- $I: L \rightarrow C(X)$.

\subsection{Bisimulation between $S W P N$ and $S W A_{\text {Reach }}$}

Definition 5. Let $Q_{N}$ be the set of states of $S W P N N$ and $Q_{A}$ the set of states of the reachable stopwatch automaton $A$. Let $R \subset Q_{N} \times Q_{A}$ be the relation between a state of the Stopwatch automaton and a state of the Stopwatch Petri Net defined by:

$\left\{\begin{array}{l}\forall(M, v) \in Q_{N} \\ \forall(l, \bar{v}) \in Q_{A}\end{array},(M, v) R(l, \bar{v}) \Leftrightarrow\left\{\begin{array}{r}M=\mathbb{M}(l) \\ v=\bar{v}\end{array}\right.\right.$

where $\mathbb{M}$ is the function giving the associated marking of a $S W A$ state $l$.

Theorem 1. $R$ is a bisimulation: For all $(M, v)$, $(l, \bar{v})$ such that $(M, v) R(l, \bar{v})$ :

$$
\begin{gathered}
\bullet(M, v) \stackrel{t_{i}}{\longrightarrow}\left(M^{\prime}, v^{\prime}\right) \Leftrightarrow\left\{\begin{array}{l}
(l, \bar{v}) \stackrel{t_{i}}{\longrightarrow}\left(l^{\prime}, \bar{v}^{\prime}\right) \\
\left(M^{\prime}, v^{\prime}\right) R\left(l^{\prime}, \bar{v}^{\prime}\right)
\end{array}\right. \\
\bullet(M, v) \stackrel{d}{\longrightarrow}\left(M, v^{\prime}\right) \Leftrightarrow\left\{\begin{array}{c}
(l, \bar{v}) \stackrel{d}{\longrightarrow}\left(l, \bar{v}^{\prime}\right) \\
\left(M, v^{\prime}\right) R\left(l, \bar{v}^{\prime}\right) \square
\end{array}\right.
\end{gathered}
$$

The proof is detailed in (Allahham and Alla (2006)).

\section{AN ILLUSTRATIVE EXAMPLE}

The priority problem in case of a sharing resource is considered in an example. Figure 3 represents the manufacturing process of two type of production $S_{1}$ and $S_{2}$ using two machines $M_{a}, M_{b}$ and a sharing robot between $S_{1}$ and $S_{2}$. The token in $P_{1}$ means that machine $M_{a}$ is operational and executes the task $1-1$ on $S_{1}$ : transition $t_{1}$ can be fired and the interval $[2,2]$ represents the duration of task 1-1. Similarly, interval $[5,7]$ represents the necessary duration for the robot to execute task $1-2$ on $S_{1}$. Machine $M_{b}$ executes task $2-1$ on $S_{2}$. [3,3] represents the duration to execute this task on $S_{2}$. Robot executes also the task $2-2$ on $S_{2}$ and the duration for that is given by $[3,4]$. We suppose here that the task robot on $S_{2}$ has priority over the the task robot on $S_{1}$. This is modeled in the following way. If $M_{b}$ finishes task $2-1$ on $S_{2}$ while the robot is executing task $1-2$ on $S_{1}$, the robot stops task $1-2$ on $S_{1}$ and executes immediately task $2-2$ on $S_{2}$. When 


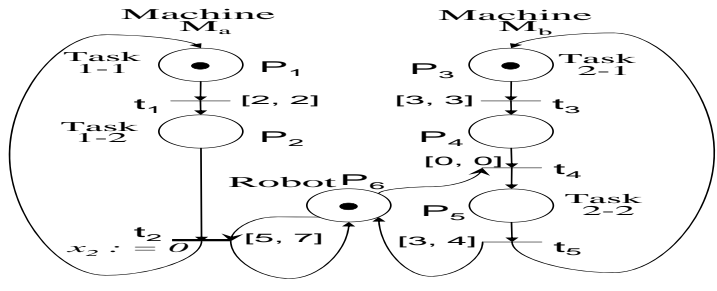

Fig. 3. $S W P N$ of two tasks with different priority on one resource

it finishes task $2-2$, task $1-2$ on $S_{1}$ is resumed by robot exactly at the point where it was stopped. This behavior is modeled in Figure 3, where only transition $t_{2}$ associated with the assignment $x_{2}:=$ 0 is interruptible. Figure 4 shows the stopwatch automaton corresponding to the $S W P N$ in Figure 3 . This is obtained applying the algorithm presented in Section 4 where the unstable state corresponding to transition $t_{4}$ has been suppressed. The Forward analysis has been executed using the model-checker (PHAVer Frehse (2005)). It is then possible to verify many properties of the system. For example, we can evaluate the system performances, especially those concerning machine $M_{a}$. It appears interesting to calculate the maximal duration where $M_{a}$ remains inactive. For that, we take advantage of stopwatch automaton and introduce a variable $y$. It is intended to calculate the duration which separates the firing of transitions $t_{1}$ and $t_{2}$. Figure 5 shows the reachable state space for the variable $y$ over all the locations of the automaton. We note that the maximum duration for which machine $M_{a}$ is remaining inactive, is $y=19$. Seeking properties depend on the system under study, our global approach gives all the needed formal results to achieve this goal.

\section{CONCLUSION}

Post and Pre-initialized Petri Nets $S W P N$ extend T-time Petri nets to allowing the formal modeling of preemption-resume behavior. We have given a method for timing analysis of this $S W P N$. This

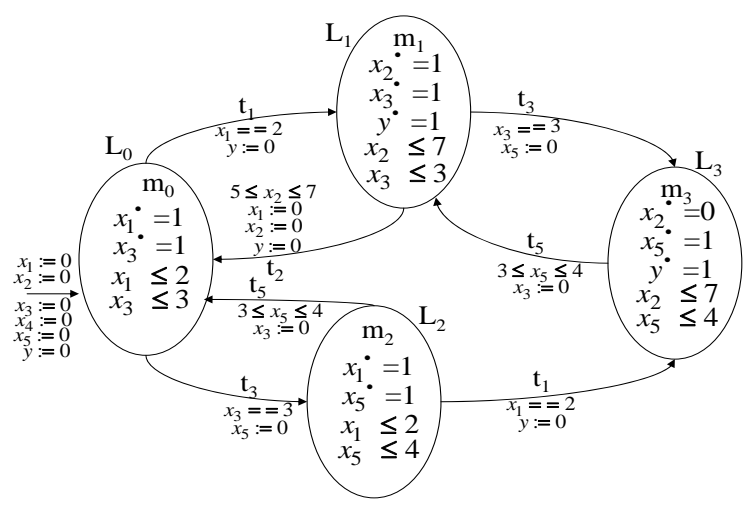

Fig. 4. Stopwatch Automaton of $S W P N$ given in Figure 3 includes a labeling algorithm of the marking graph to build a Stopwatch Automaton $S W A_{G}$. Then, a forward computation of the state space is executed by PHAVer model-checker. The resulting stopwatch automaton and $S W P N$ are timed bisimilar.

\section{REFERENCES}

A. Allahham and H. Alla. Monitoring the interruptible discrete events systems. Interne raport, Laboratoire d'Automatique de Grenoble - INPG, France, 2006.

R. Alur, C. Courcoubetis, N. Halbwachs, T.A. Henzingerd, P.H. Hod, X. Nicollin, A. Olivero, J. Sifakis, and S. Yovine. The algoritmic analysis of hybrid systems. Theoretical Computer Science, 138(1), 1995.

B. Berthomieu and M. Diaz. Modeling and verification of time dependent systems using time petri nets. IEEE Transaction Software Engineering, 17(3), 1991.

Andrea Bobbio, Antonio Puliafito, and Miklós Telek. A modeling framework to implement preemption policies in non-Markovian SPNs. IEEE Transactions on Software Engineering, 26 (1):36-54, January 2000.

G. Bucci, A. Fedeli, L. Sassoli, and E. Vicario. Timed state space analysis of real-time preemptive systems. IEEE Transactions on Software engineering, 30(2):97-111, 2004.

F. Cassez and K.J. Larsen. The impressive power of stopwatch. pages 138-152, 2000.

R. David and H. Alla. Discrete, Continuous and Hybrid Petri Nets. Springer, 2005.

G. Frehse. Phaver: Algorithmic verification of hybrid systems past hytech. In The Fifth International Workshop on Hybrid Systems: Computation and Control, pages 258-273, 2005.

D. Lime and O. H. Roux. A translation based method for the timed analysis of scheduling extended time petri nets. In 25th IEEE International Real time Systems Symposuim, pages 187-196, Lisbon, Portugal, 2004.

O. H. Roux and D. Lime. Time petri nets with inhibitor hyperarcs. formal semantics and state space computation. In 25th International Conference on theory and application of Petri nets, pages 371-390, Bologna, Italy, 2004.

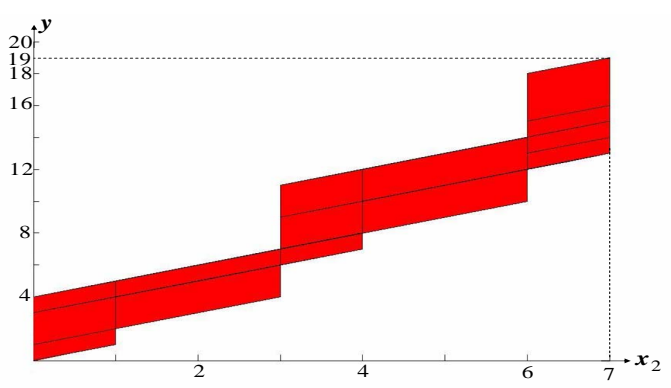

Fig. 5. Reachable space state of the variable $y$ 